\title{
A pilot feasibility study of daily rTMS to modify corticospinal excitability during lower limb immobilization
}

\author{
Raffaella Ricci ${ }^{2}$ \\ Dave Ramsey ${ }^{3}$ \\ Kevin Johnson' \\ Jeffrey J Borckardt' \\ Matthew Vallejo' \\ Donna R Roberts ${ }^{4}$ \\ Mark S George'
}

'Brain Stimulation Laboratory (BSL), Institute of Psychiatry; ${ }^{4}$ Department of Radiology, Medical University of South Carolina, Charleston, SC, USA; ${ }^{2}$ Department of Psychology, University of Turin, Turin, Italy; ${ }^{3}$ South Carolina Research Authority (SCRA), Columbia, SC, USA

Correspondence: Raffaella Ricci Brain Stimulation Laboratory, MUSC IOP, 502 N, 67 President Street, Charleston SC 29425 , USA

Tel + I 8437926922

Fax +I 8437925702

Email ricci@musc.edu

\begin{abstract}
Short term immobilization of the lower limb is associated with increased corticospinal excitability at 24 hours post cast removal. We wondered whether daily stimulation of the motor cortex might decrease brain reorganization during casting. We tested the feasibility of this approach. Using transcranial magnetic stimulation (TMS), resting motor threshold and recruitment curves were obtained at baseline in 6 healthy participants who then had leg casts placed for 10 days. On 7 of the 10 days subjects received 20 minutes of $1 \mathrm{~Hz}$ repetitive TMS (rTMS). TMS measures were then recorded immediately after and 24 hours post cast removal. Four of 6 subjects completed the study. At the group level there were no changes in excitability following cast removal. At the individual level, two participants did not show any change, 1 participant had higher and one lower excitability 24 hours after cast removal. Daily rTMS over motor cortex is feasible during casting and may modify neuroplastic changes occurring during limb disuse. A prospective double blind study is warranted to test whether daily rTMS might improve outcome in subjects undergoing casting, and perhaps in other forms of limb disuse such as those following brain injury or weightlessness in space flight.
\end{abstract}

Keywords: motor cortex, plasticity, rTMS, limb disuse, motor recovery

\section{Introduction}

Lower limb disuse has been employed as a model to investigate motor cortex plasticity consequent to altered motor functioning (Roberts et al 2007). We wondered whether it was feasible and safe to use transcranial magnetic stimulation (TMS) to potentially modify or prevent motor cortex reorganization consequent to limb disuse. Neuronal plasticity refers to functional changes within the central nervous system outlasting specific internally or externally induced manipulations (Classen et al 2003). Brain plasticity underlies the formation of new motor skills (Pascual-Leone et al 1995; Classen et al 1998; Lotze et al 2003; Perez et al 2004) as well as cortical reorganization after damage to the central nervous system (Nudo et al 2001; Ward 2005) or sensory deafferentation (Chen 1998; Ziemann et al 1998; Cohen et al 1991a, 1991b; Werhahn et al 2002). It has been shown that transient limb nonuse, such as that occurring during limb immobilization (Liepert et al 1995; Zanette et al 1997, 2004), induces functional changes in the motor cortex.

Neuronal plasticity likely plays an adaptive role following brain injury or when subjects learn new skills or encounter new environments. However, during long periods of limb disuse such as during prolonged bedrest or wearing a cast, the brain's reorganization may cause motor impairment when attempting to return to normal functioning. Also in patients suffering from brain injury, functional brain changes related to limb nonuse might interfere with their recovery.

In a previous study in 8 healthy volunteers (Roberts et al 2007), we used TMS to measure neuroplastic changes occurring after 10 days of wearing a full leg cast. 
Motor cortex excitability was determined immediately after and 24 hours after cast removal and compared with baseline values. Eight other subjects did not wear a cast and served as a control group. Corticospinal excitability over the affected leg area was found to be maximal 24 hours after cast removal, as indexed by an increased slope of the recruitment curve. The control group (no cast) did not have excitability changes over the same timeframe and location. In that study, the increased excitability of the corticospinal pathway likely played a role in recovering and returning the brain to its precasting state. For example, cortical excitability increases during acquisition of a new or novel motor task or as a consequence of motor skill training (Lotze et al 2003; Perez et al 2004).

Since low frequency TMS can cause cortical inhibition (Chen et al 1997; Muellbacher et al 2000), we wondered whether daily repetitive TMS (rTMS) over the motor cortex involving the casted leg might decrease the brain changes during casting, as indexed by increased excitability after cast removal (Roberts et al 2007). Alternatively, the TMS activation of motor output fibers, and perhaps reflex sensory input, would stop brain reorganization. TMS might serve as an artificial 'practice' of normal movement during the casting period, inhibiting reorganization.

For both the above scenarios, we expect for rTMS to prevent the cortical changes which otherwise would occur as adaptive response to limb nonuse, during the immobilization, in the brain. As a consequence of daily rTMS treatment, we expect to observe no signs of motor re-learning (reflected by increased cortical excitability), when the cast is removed and the limb is re-used.

Transcranial magnetic stimulation is a safe and noninvasive technique for exploring brain representation and cortical plasticity (Cohen et al 1998; Siebner et al 2003). Daily rTMS over prefrontal cortex for several weeks has antidepressant effects (George et al 1995, 1996, 1999). Its potential benefits to the application of other brain pathologies, such as Parkinson disease (Sommer et al 1996), pain syndromes (Borckardt et al 2006), and cortical epilepsy (Tergau et al 1999; Epstein et al 1999), are under evaluation (Epstein et al 2003; Gow et al 2003). In stroke patients, some studies of slow rTMS over the unaffected hemisphere have found improvement in symptoms such as unilateral neglect (Oliveri et al 2001; Brighina et al 2003), aphasia (Martin et al 2004; Naeser et al 2005a, 2005b) and motor performance (Mansur et al 2005; Takeuchi et al 2005). Recent studies also show that high frequency rTMS over the damaged hemisphere enhances corticospinal excitability (Di Lazzaro et al 2006) and improves motor performance (Khedr et al 2005; Kim et al 2006) in patients with stroke.
In this initial open pilot feasibility trial, we applied rTMS over the leg motor area as a potential 'treatment' during the period of leg immobilization. Low frequency rTMS was chosen because of safety reasons and because it causes transient cortical inhibition (Chen et al 1997; Wassermann et al 1998; Muellbacher et al 2000). This safety measure was particularly important in our experimental protocol since the motor cortex is known to be highly susceptible to TMS-induced seizures. We also used TMS to quantify the degree of cortical reorganization caused by the effects of lower limb immobilization and TMS treatment. TMS measures of motor cortex excitability for the left gastrocnemius and ipsilateral abductor pollicis brevis (APB) were gathered before, immediately after and 24 hours after cast removal. Therefore, in this study TMS was used as both a tool to investigate cortical excitability changes and as a potential 'intervention' or treatment.

\section{Methods}

\section{Subjects}

Six right-handed healthy volunteers (4 women and 2 men) were enrolled. Subjects gave their written informed consent to participate in this study, which was approved by the Medical University of South Carolina Institutional Review Board (IRB). Two women dropped out of the study after 3-4 days of limb immobilization because of discomfort caused by the cast. Therefore, only 4 out of 6 participants completed the full study. The four participants had a mean age of 25.3 years (range 18-34) and an educational level of 14.5 (range 12-16) years. On the first day of the study, participants were screened against inclusion/exclusion criteria and a physical exam was performed by a licensed physician (MSG). Since the most well-known safety concern of TMS is a seizure, potential participants who had a history of epilepsy or intracranial abnormality were not included.

\section{TMS protocol}

Stimuli were delivered through a MagStim Super Rapid TMS machine (The Magstim Company Limited, Whitland, South West Wales, UK). A flat figure-eight coil, with each wing about $9 \mathrm{~cm}$ in diameter, was used to measure cortical excitability from the hand motor area. A larger, butterfly shaped coil, in which each wing had an outer diameter of $13 \mathrm{~cm}$, was used for measuring cortical excitability of the leg. This same large coil was used for the daily 'treatments'. We used 2 different large coils because of a heating problem. When a coil became too hot the session was paused, the coil was switched, and then the session resumed. 


\section{Recording procedure}

During TMS sessions, subjects were asked to lie comfortably supine with their lower extremities fully relaxed. Surface electrodes were placed along the medial gastrocnemius muscle bellies bilaterally. For recording of motor evoked potentials (MEPs), surface electrodes were placed over the belly of the left gastrocnemius. Also measures of motor cortex excitability were recorded from the left APB muscles in a belly-tendon montage. The APB served as a control region since it was expected that subjects continued to use the upper extremities in the usual fashion throughout the lower limb casting period. MEPs were recorded using equipment and software from Cambridge Electronic Design (Cambridge, England, UK), the signal was converted from analogue to digital using the Micro 1401 MK II (Cambridge Electronic Design) and conditioned using the CED1902 signal conditioner (Cambridge Electronic Design). The signal was sampled for $1000 \mathrm{msec}$ at a rate of $5 \mathrm{KHz}$ and amplified with a gain of 1000. Sampling began $10 \mathrm{msec}$ before the TMS pulse was delivered and a marker was written to the file with each TMS pulse using a 10-msec 5-volt transitor-transitor logic signal that was edge-gated from low to high. The analog filter was set with a high pass of $0.5 \mathrm{~Hz}$ and a low pass of $1000 \mathrm{~Hz}$. Alternating current coupling was applied. To further condition the signal, digital filtering was applied to remove $60 \mathrm{~Hz}$ noise and a high-pass filter with corner frequency of $30 \mathrm{~Hz}$ and transition gap of $13 \mathrm{~Hz}$ was used. For each of the two muscles, the optimal location for stimulus induction (the location that gives the maximum MEP amplitude for the specific muscle being investigated) was identified.

\section{TMS measures of cortical excitability}

Resting motor threshold (rMT) and recruitment curves (RC) were recorded for the left gastrocnemius and the left APB muscles, through the computer program Spike 2 using surface EMG. The rMT was defined as the minimal stimulus intensity that is required to achieve an MEP from the target muscle of at least $50 \mu \mathrm{V}$. rMT was determined using EMG recording and the PEST procedure (Mishory et al 2004). Resting motor threshold was gathered twice to ensure a more stable measure (the first subject [A] underwent only one rMT measurement). RC related stimulation intensity to MEP amplitude. Six stimuli were delivered at each of a series of 6 different intensities starting 5 points below the rMT and increasing by 5 increments ( $\%$ maximum machine output: $-5,0,+5,+10,+15,+20)$. This procedure was performed during rest. The slope of the recruitment curve is a measure of corticospinal excitability with increasing slope indicating increased excitability (Devanne et al 1997). These procedures were repeated for each of the two muscles (APB first) during each TMS investigative session (pre-cast, post-cast, 24 hours post-cast), but were not done on the days of TMS treatment.

\section{Experimental paradigm}

All participants underwent identical TMS procedures before and after the 10 day casting period. A TMS excitability measurement was also acquired 24 hours after cast removal. After the first TMS evaluation, they were taken to the MUSC hospital cast room where their left lower limb was immobilized by a full leg cast (open at the level of the toes and extending to mid thigh). Participants were previously provided with crutches and briefly trained on how to use them. As in the previous study (Roberts et al 2007), participants wore the cast for 10 days. However, in this study they also received a 20 minute $1 \mathrm{~Hz}$ rTMS treatment on 7 out of the 10 days of casting. rTMS was applied on the scalp over the motor cortex spot able to elicit a leg movement at the motor threshold intensity. The leg area was re-determined each day before starting the treatment. Once the leg area was found, the coil was immobilized using a holder and the intensity was set at $90 \%$ of each person's leg resting motor threshold, which was determined with the EMG method (Pridmore et al 1998). In two subjects (A and C) this intensity was found to induce an overt movement on the first day of treatment. This was likely due to fluctuations of resting MT values. Therefore, for them we used an intensity of $90 \%$ of visual rMT which corresponded to $74 \%$ and $80 \%$ of rMT (as determined with the EMG method). On the tenth day, after removing the cast, the participants were brought to the TMS room in a wheelchair to avoid the impact of limb use on the TMS measurements. A final TMS examination was performed 24 hours after cast removal (see Figure 1). Each TMS session took less than 1 hour and occurred approximately at the same time of day.

\section{Data analysis}

Resting motor thresholds for the leg and separately for the hand were analyzed for the subjects, as a group, using the nonparametric Friedman test.

Hierarchical linear modeling (HLM) was used to assess the effects of visit (1 through 3 ) on individual recruitment curves. We used this analysis for the following two reasons: 1) to make our results easier to compare with those of the previous study (Roberts et al 2007); and 2) because HLM allows for modeling of variables at the individual 


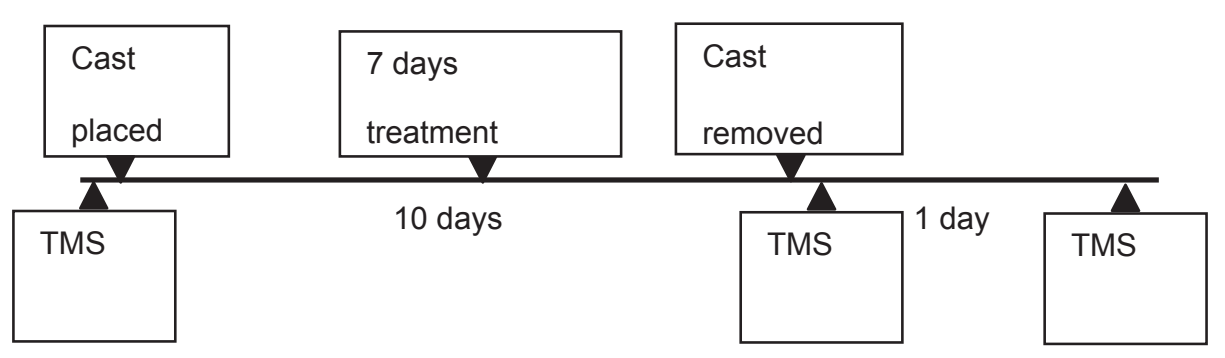

Figure I Time course of the experiment.

Abbreviations: TMS, transcranial magnetic stimulation.

subject-level (eg, each subject's individual RCs). MEP values that comprised the RCs were log-transformed to correct for nonlinearity and nonnormality of the curves for statistical analyses. The estimation method of the model was restricted maximum likelihood (REML) and the covariance structure was "Unstructured." Two separate analyses were performed for 'leg' and 'hand' since two different coils were used to stimulate the motor cortex.

\section{Results}

\section{Resting motor thresholds}

Resting motor thresholds from the leg and hand motor area for each participant are reported in Table 1. For the subjects, as a group, the nonparametric Friedman test did not show any significant difference between rMT across conditions, for the leg and separately for the hand.

\section{Recruitment curves}

\section{Leg}

Participants as a group did not show any significant effects of the visit $(\mathrm{F}[2,440]=0.07$, ns) on RC slope. At the individual level, two participants did not show any significant effect of the visit while the other two showed significant differences between visit 1 and 3, in the opposite directions. Subject $\mathrm{C}$ evidenced an increase in RC slope in the casted leg from visit 1 to visit $3(\mathrm{~F}[2,102]=4.31, \mathrm{p}=0.0159)$ while subject $\mathrm{B}$ evidenced a decrease in RC slope in the casted leg from visit 1 to visit $3(\mathrm{~F}[2,102]=3.05, \mathrm{p}=0.0517)$. No other differences were observed between visits (see Figure 2).

\section{Hand}

Participants as a group did not show any significant effects of the visit $(\mathrm{F}[2,423]=2.25$, ns) on recruitment curve slope. At the individual level, two participants did not have any significant effect of 'visit' while the other two showed significant differences between visit 3 and 2. Subject A evidenced an increase in RC slope in the hand from visit 2 to visit 3 $(\mathrm{F}[2,102]=5.68, \mathrm{p}=0.0046)$ while subject $\mathrm{D}$ evidenced $\mathrm{a}$ decrease in RC slope from visit 2 to visit $3(\mathrm{~F}[2,102]=11.27$, $\mathrm{p}<0.0001)$. No other differences in RC slope were observed between visits.

\section{Side effects, tolerability, dropouts}

Relatively common side effects of TMS are a muscle tension type headache and discomfort at the site of stimulation (Anderson et al 2006). The participants of this study were asked soon after and before starting with the next session whether they experienced headache or discomfort due to the previous TMS session (both for the measurements and the treatments). None of them reported any of the above symptoms.

The risks of 10 days of leg casting included chafing, skin irritation, pain and discomfort, itching, and reduced mobility.

Table I Individual resting motor thresholds (rMTs), Mean rMTs and relative standard deviations (SDs) recorded from the leg and hand motor areas, for the different transcranial magnetic stimulation conditions

\begin{tabular}{|c|c|c|c|c|c|c|}
\hline & \multicolumn{3}{|l|}{ Leg } & \multicolumn{3}{|l|}{ Hand } \\
\hline & Pre-cast & Post-cast & 24 Post & Pre-cast & Post-cast & 24 Post \\
\hline$A$ & 74 & 65 & 64 & 70 & 65 & 62 \\
\hline B & 43 & 49 & 47 & 49 & 54 & 59 \\
\hline C & 47 & 45 & 53 & 58 & 60 & 56 \\
\hline D & 74 & 72 & 68 & 92 & 80 & 85 \\
\hline Mean (SD) & $57(15.7)$ & $58(\mathrm{II} .7)$ & 58 (9.09) & $67(18.8)$ & $65(10.1)$ & $65(12.4)$ \\
\hline
\end{tabular}


Participant - A

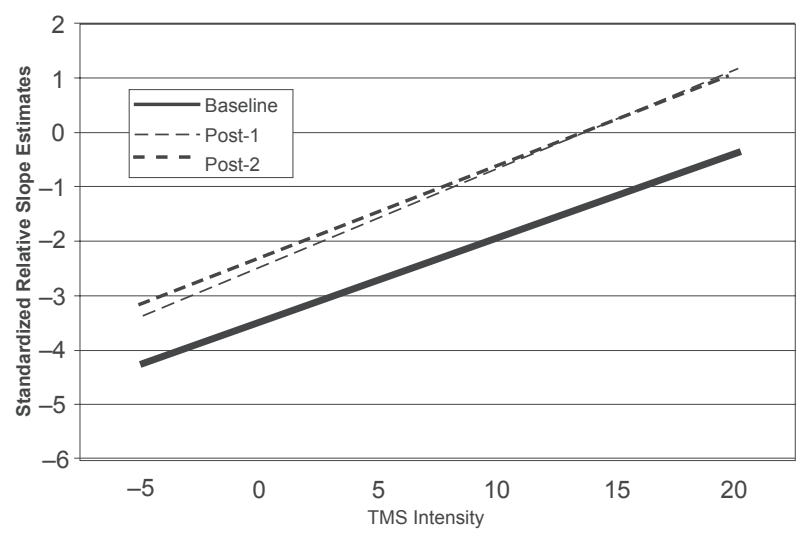

Participant - C

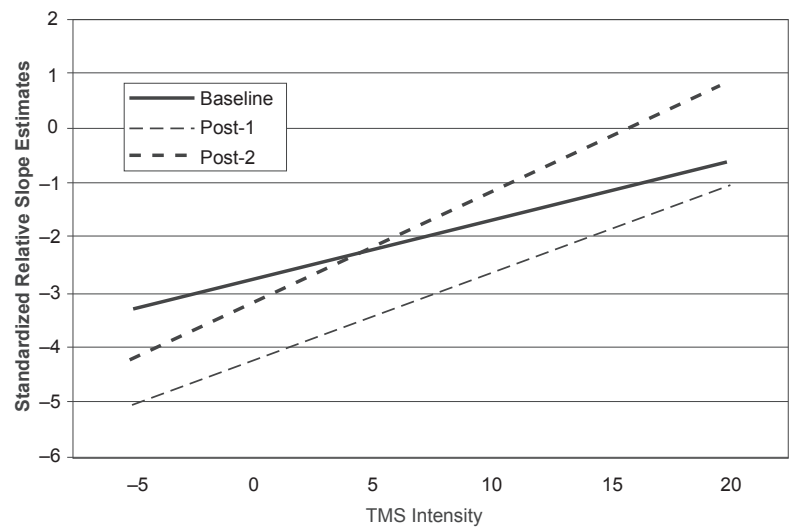

Participant - B

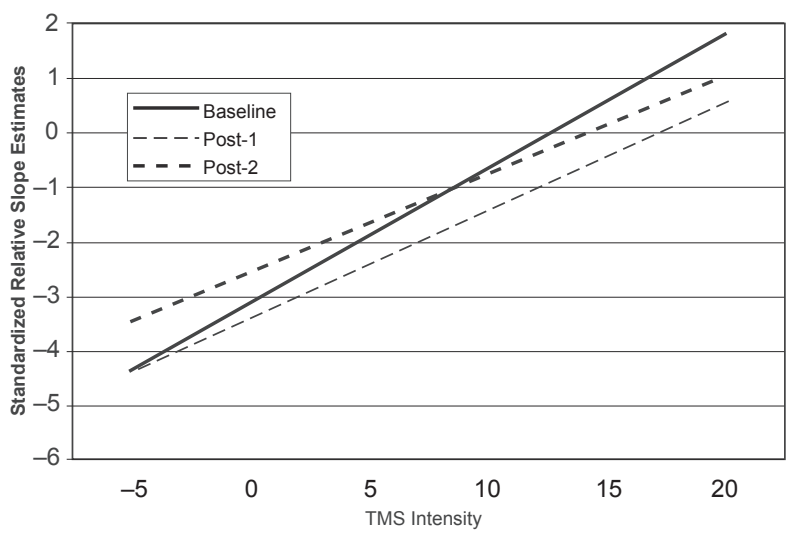

Participant - D

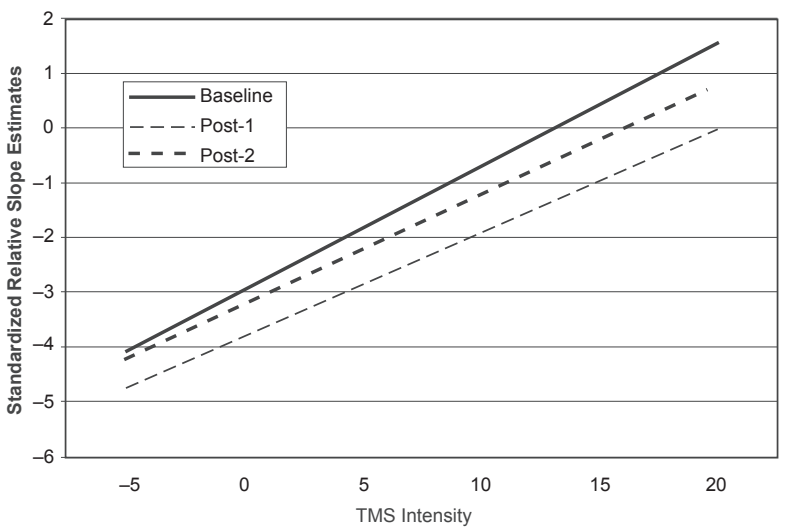

Figure 2 Recruitment curves (RC) of the left leg for each individual participant (A, B, C, and D). Standardized relative RC slope estimates for the left leg of each individual as a function of transcranial magnetic stimulation (TMS) level for each of the three visits. No significant differences were found in curve slope between visits for participants $\mathbf{A}$ and $\mathbf{C}$, while RC slope decreased for participant $\mathbf{B}$ and increased for participant $\mathbf{C}$.

10 days of leg casting was not expected to produce any prominent muscle atrophy on the basis of the previous study (Roberts et al 2007). When participants were asked about side effects of the cast all of them reported some discomfort from wearing the cast. As previously mentioned, two out of the six participants who first entered the study, dropped out after a few days because of discomfort due to the cast. However, as in the previous study (Roberts et al 2007), also in our investigation, wearing a full leg cast for 10 days did not produce any serious consequences (such as clotting) in any of our healthy participants.

\section{Discussion and conclusions}

Long periods of limb disuse can lead to motor impairments (Antonutto et al 1998; Fitts et al 2000) which are not completely explained by changes of the musculoskeletal system but rather also depend on functional changes within the motor cortex (Liepert et al 1995; Zanette et al 1997). TMS has been used to detect subtle changes of corticospinal excitability occurring after a very short period of limb immobilization (Roberts et al 2007). With the present study, we investigated the feasibility and safety of daily TMS as a technique for preventing and/or reducing the effects of limb disuse on the central nervous system.

Previous results (Roberts et al 2007) showed that returning to normal activity after 10 days of wearing a lower limb cast requires a "relearning" period, which is associated with high corticospinal excitability, peaking at 24 hours after cast removal. In the present study, participants were treated with 20 minutes of $1 \mathrm{~Hz}$ rTMS on 7 out of 10 days of casting. Contrary to our earlier casting study where daily TMS was not administered (Roberts et al 2007), the participants as a group in the current study did not show any change in corticospinal excitability across conditions. At a single 
case analysis, only 1 of the 4 subjects in this preliminary trial evidenced an increase in RC slope 24 hours following cast removal. While it is certainly plausible that the lack of observed effect for the group is due to low statistical power, it is also possible that stimulation of the motor cortex during casting led to no observable change in MEP curves post castremoval. As we mentioned in the methods, HLM allows one to analyze data at the individual subject-level. Twenty-four hours after removing the cast, RC slopes for the leg area did not change in 2 participants, were enhanced in 1 participant and decreased in another one. This may explain why at a group level post-cast cortical excitability measures did not differ from baseline values. At least in some individuals, stimulation of the motor cortex during casting might have reduced cortical excitability changes occurring in response to limb immobilization (Liepert et al 1995; Zanette et al 1997, 2004; Roberts et al 2007). Discrepancies of TMS effects among subjects can be explained by interindividual variability of rTMS on cortical excitability (Maeda et al 2000a), differences in stimuli intensity (for subject $\mathrm{C}$ we had to use $80 \%$ instead of $90 \%$ of the original rMT), or other factors (ie, variability of subjects in following the instruction of minimizing the use of the casted leg).

Importantly for the aim of this feasibility study, none of our healthy participants reported any of the common symptoms (muscle tension type headache and discomfort at the site of stimulation) due to the TMS sessions (both for the measurements and the daily rTMS treatments). In addition, as in the previous study (Roberts et al 2007), 10 days of leg casting did not produce any serious consequences (such as clotting) in any of our participants. However, when participants were asked about their experience and possible side effects of wearing the cast, all of them reported some discomfort. This was reflected by the high rate of drop out (two out of six participants) observed after few days of casting and due to discomfort by wearing the cast. This result need to be kept in mind for planning future immobilization studies requiring casting of the lower limb.

We have shown that this type of research is feasible. Interpretations of the mechanisms underlying the possible effects of rTMS on changes of motor cortex excitability following casting are too speculative at this point (considering this is a feasibility and small sample size study). However, we like to advance some tentative interpretations in relation to our previous findings (Roberts et al 2007). One explanation for our failure to find post-casting increases in cortical excitability, although admittedly soft, is that daily TMS inhibited the motor cortex from reorganizing throughout a period of time during which, because of limb disuse, it would otherwise have undergone functional changes. Multiple applications of rTMS over cortical regions that would otherwise have reorganized might have produced a greater influence in reducing neuroplasticity as suggested by the evidence that the rTMS effect increases the second day of stimulation (Maeda et al 2000b). If our interpretation is correct, the question remains of whether the mechanism underlying rTMS' ability to impede reorganization is similar to that underlying passive limb movements, imagined activity, or peripheral nerve stimulation, or rather must be assimilated to brain activation occurring during voluntary limb movements (Bohning et al 2000; Li et al 2004).

Low frequency TMS has been shown to transiently decrease cortical excitability (Chen et al 1997; Wassermann et al 1998). In stroke patients daily 1 Hz rTMS treatment over the unaffected hemisphere ameliorates symptoms such as aphasia or unilateral neglect (Oliveri et al 2001; Brighina et al 2003; Martin et al 2004; Naeser et al 2005a, 2005b) and, even more important for the topic of this study, motor disorders (Mansur et al 2005; Takeuchi et al 2005). Transcranial direct current stimulation (Brown et al 2003; Hummel et al 2005; Stong 2006), dual stimulation (TMS and peripheral nerve stimulation) (Uy et al 2003), and high frequency magnetic stimulation (Khedr et al 2005; Kim et al 2006) of the affected motor cortex have been shown to improve hemiparesis after stroke. Even though these studies showed safety and efficacy of stimulation of the damaged hemisphere, computer modeling data (Wagner et al 2006) indicates the risks of using conventional standards when applying TMS proximally to a lesion site (where tissue geometry and conductivity are altered by the stroke). However, in a nondamaged brain which undergoes neuroplastic changes in response to limb disuse (as in orthopedic patients) it might be more effective directly treating the motor cortex contralateral to the inactive limb. This should be particularly true when the treatment starts at the beginning of the period of limb disuse.

Studies on neurological patients indicate functional motor improvement by magnetic (Khedr et al 2005; Mansur et al 2005; Takeuchi et al 2005; Kim et al 2006) or electric brain stimulation (Brown et al 2003). Our findings suggest that daily rTMS is feasible and safe, and may reduce neuroplastic changes of cortical excitability consequent to casting (Roberts et al 2007). These pilot data need to be further investigated in a randomized double blind study in which also corticospinal excitability from the nonimmobilized limb needs to be evaluated, as a control condition. If these results are confirmed, repeated rTMS might be used as a preventive 
treatment in orthopedic patients with limb immobilization and in other forms of limb disuse such as those following brain injury or weightlessness in space flight.

\section{Acknowledgments}

This study was funded by NASA - Epscor award number NCC5/575. The Brain Stimulation Laboratory is supported in part by the Stanley Foundation, the National Alliance for Research on Schizophrenia and Depression (NARSAD), NINDS grant RO1-AG40956, NIMH grant RO1 MH069887, 1K08MH070915-01A1 (Nahas), and 1K23NS050485-01A2 (Borckardt). The authors would like to thank Minnie Dobbins for administrative help.

\section{References}

Anderson B, Mishory A, Nahas Z, et al. 2006. Tolerability and safety of high daily doses of repetitive transcranial magnetic stimulation in healthy young men. J Ect, 22:49-53.

Antonutto G, Bodem G, Zamparo P, et al. 1998. Maximal power and EMG of lower limbs after 21 days spaceflight in one astronaut. J Gravitational Physiology, 5:63-6.

Bohning DE, Shastri A, McGavin L, et al. 2000. Motor cortex brain activity induced by $1-\mathrm{Hz}$ transcranial magnetic stimulation is similar in location and level to that for volitional movement. Invest Radiol, 35:676-83.

Borckardt JJ, Weinstein M, Reeves ST, et al. 2006. Postoperative left prefrontal repetitive transcranial magnetic stimulation reduces patientcontrolled analgesia use. Anesthesiology, 105:557-62.

Brighina F, Bisiach E, Oliveri M, et al. 2003. $1 \mathrm{~Hz}$ repetitive transcranial magnetic stimulation of the unaffected hemisphere ameliorates contralesional visuospatial neglect in humans. Neurosci Lett, 336:131-3.

Brown JA, Lutsep H, Cramer SC, et al. 2003. Motor cortex stimulation for enhancement of recovery after stroke: case report. Neurol Res, $25: 815-18$

Chen R, Classen J, Gerloff C, et al. 1997. Depression of motor cortex excitability by low-frequency transcranial magnetic stimulation. Neurology, 48:1398-403.

Chen R, Corwell B, Yaseen Z, et al. 1998. Mechanisms of cortical reorganization in lower-limb amputees. J Neurosci, 18:3443-50.

Classen J, Liepert J, Wise SP, et al. 1998. Rapid plasticity of human cortical movement representation induced by practice. $J$ Neurophysiol, 79:1117-23.

Classen J, Ziemann U. 2003. Stimulation-induced plasticity in the human motor cortex. In: Boniface S, Ziemann U (eds). Plasticity in the Human Nervous System. Cambridge: Cambridge University Press, pp. $135-65$.

Cohen LG, Bandinelli S, Findley TW, et al. 1991. Motor reorganization after upper limb amputation in man. A study with focal magnetic stimulation. Brain, 114:615-27.

Cohen LG, Bandinelli S, Topka HR, et al. 1991. Topographic maps of human motor cortex in normal and pathological conditions: mirror movements, amputations and spinal cord injuries. Electroencephalogr Clin Neurophysiol Suppl, 43:36-50.

Cohen LG, Ziemann U, Chen R, et al. 1998. Studies of neuroplasticity with transcranial magnetic stimulation. J Clin Neurophysiol, 15:305-24.

Devanne H, Lavoie BA, Capaday C. 1997. Input-output properties and gain changes in the human corticospinal pathway. Exp Brain Res, 114:329-38.

Di Lazzaro V, Dileone M, Profice P, et al. 2006. Direct demonstration that repetitive transcranial magnetic stimulation can enhance corticospinal excitability in stroke. Stroke, 37:2850-3.
Epstein CM, Rothwell JC. 2003. Therapeutic uses of rTMS. In: Boniface S, Ziemann U (eds). Plasticity in the Human Nervous System. Cambridge: Cambridge University Press, pp. 246-63.

Epstein CM. 2005. TMS in Epilepsy. In: George MS, Belmaker RH (eds). Transcranial Magnetic Stimulation in Neuropsychiatry. Washington, DC: American Psychiatric Press, Inc., pp. 172-84.

Fitts RH, Riley DR, Widrick JJ. 2000. Physiology of a microgravity environment invited review: microgravity and skeletal muscle. J Applied Physiology, 89:823-39.

George MS, Lisanby SH, Sackeim HA. 1999. Transcranial magnetic stimulation: applications in neuropsychiatry. Arch Gen Psychiatry, 56:300-11.

George MS, Wassermann EM, Williams WA, et al. 1995. Daily repetitive transcranial magnetic stimulation (rTMS) improves mood in depression. Neuroreport, 6:1853-6.

George MS, Wassermann EM, Williams WA, et al. 1996. Changes in mood and hormone levels after rapid-rate transcranial magnetic stimulation (rTMS) of the prefrontal cortex. J Neuropsychiatry Clin Neurosci, 8:172-80.

Gow D, Fraser C, Hamdy S. 2003. Rehabilitation. In: Boniface S, Ziemann U (eds). Plasticity in the Human Nervous System. Cambridge: Cambridge University Press, pp. 264-7.

Hummel F, Celnik P, Giraux P, et al. 2005. Effects of non-invasive cortical stimulation on skilled motor function in chronic stroke. Brain. 128:490-9.

Khedr EM, Ahmed MA, Fathy N, et al. 2005. Therapeutic trial of repetitive transcranial magnetic stimulation after acute ischemic stroke. Neurology, 65:466-8.

Kim YH, You SH, Ko MH, et al. 2006. Repetitive transcranial magnetic stimulation-induced corticomotor excitability and associated motor skill acquisition in chronic stroke. Stroke, 37:1471-6.

Li X, Teneback CC, Nahas Z, et al. 2004. Interleaved transcranial magnetic stimulation/functional MRI confirms that lamotrigine inhibits cortical excitability in healthy young men. Neuropsychopharmacology, 29:1395-407.

Liepert J, Tegenthoff M, Malin JP. 1995. Changes of cortical motor area size during immobilization. Electroencephalogr Clin Neurophysiol, 97:382-6.

Lotze M, Braun C, Birbaumer N, et al. 2003. Motor learning elicited by voluntary drive. Brain, 126:866-72.

Maeda F, Keenan JP, Tormos JM, et al. 2000. Interindividual variability of the modulatory effects of repetitive transcranial magnetic stimulation on cortical excitability. Exp Brain Res, 133:425-30.

Maeda F, Keenan JP, Tormos JM, et al. 2000. Modulation of corticospinal excitability by repetitive transcranial magnetic stimulation. Clin $\mathrm{Neu}-$ rophysiol. 111:800-5.

Mansur CG, Fregni F, Boggio PS, et al. 2005. A sham stimulation-controlled trial of rTMS of the unaffected hemisphere in stroke patients. Neurology, 64:1802-4.

Martin PI, Naeser MA, Theoret H, et al. 2004. Transcranial magnetic stimulation as a complementary treatment for aphasia. Semin Speech Lang, 25:181-91.

Mishory A, Molnar C, Koola J, et al. 2004. The maximum-likelihood strategy for determining transcranial magnetic stimulation motor threshold, using parameter estimation by sequential testing is faster than conventional methods with similar precision. $J$ Ect, 20:160-5.

Muellbacher W, Ziemann U, Boroojerdi B, et al. 2000. Effects of lowfrequency transcranial magnetic stimulation on motor excitability and basic motor behavior. Clin Neurophysiol, 111:1002-7.

Naeser MA, Martin PI, Nicholas M, et al. 2005. Improved naming after TMS treatments in a chronic, global aphasia patient - case report. Neurocase, 11:182-93.

Naeser MA, Martin PI, Nicholas M, et al. 2005. Improved picture naming in chronic aphasia after TMS to part of right Broca's area: an openprotocol study. Brain Lang, 93:95-105.

Nudo RJ, Plautz EJ, Frost SB. 2001. Role of adaptive plasticity in recovery of function after damage to motor cortex. Muscle Nerve, 24:1000-19. 
Oliveri M, Bisiach E, Brighina F, et al. 2001. rTMS of the unaffected hemisphere transiently reduces contralesional visuospatial hemineglect. Neurology, 57:1338-40.

Pascual-Leone A, Nguyet D, Cohen LG, et al. 1995. Modulation of muscle responses evoked by transcranial magnetic stimulation during the acquisition of new fine motor skills. $J$ Neurophysiol, 74:1037-45.

Perez MA, Lungholt BK, Nyborg K, et al. 2004. Motor skill training induces changes in the excitability of the leg cortical area in healthy humans. Exp Brain Res, 159:197-205.

Pridmore S, Fernandes Filho JA, Nahas Z, et al. 1998. Motor threshold in transcranial magnetic stimulation: a comparison of a neurophysiological method and a visualization of movement method. J Ect, 14:25-7.

Roberts DR, Ricci R, Funke FW, et al. 2007. Lower limb immobilization is associated with increased corticospinal excitability. Exp Brain Res, 181:213-20.

Siebner HR, Rothwell J. 2003. Transcranial magnetic stimulation: new insights into representational cortical plasticity. Exp Brain Res, 148:1-16.

Sommer M, Tergau F, Paulus W. 2005. TMS in hypokinetic movement disorders. In: George MS, Belmaker RH (eds). Transcranial Magnetic Stimulation in Neuropsychiatry. Washington, DC: American Psychiatric Press, Inc., pp. 163-72.

Stong C. 2006. Cortical stimulation for hemiparetic stroke patients - safety and efficacy data. Neurol Rev, 13:8-11.

Takeuchi N, Chuma T, Matsuo Y, et al. 2005. Repetitive transcranial magnetic stimulation of contralesional primary motor cortex improves hand function after stroke. Stroke, 36:2681-6.
Tergau F, Naumann U, Paulus W, et al. 1999. Low-frequency repetitive transcranial magnetic stimulation improves intractable epilepsy. Lancet, 353:2209.

Uy J, Ridding MC, Hillier S, et al. 2003. Does induction of plastic change in motor cortex improve leg function after stroke? Neurology, 61:982-4.

Wagner T, Fregni F, Eden U, et al. 2006. Transcranial magnetic stimulation and stroke: A computer-based human model study. Neuroimage, 30:857-70.

Ward NS. 2005. Mechanisms underlying recovery of motor function after stroke. Postgrad Med J, 81:510-14.

Wassermann EM. 1998. Risk and safety of repetitive transcranial magnetic stimulation: report and suggested guidelines from the International Workshop on the Safety of Repetitive Transcranial Magnetic Stimulation, June 5-7, 1996. Electroencephalogr Clin Neurophysiol, 108:1-16.

Werhahn KJ, Mortensen J, Van Boven RW, et al. 2002. Enhanced tactile spatial acuity and cortical processing during acute hand deafferentation. Nat Neurosci, 5:936-8.

Zanette G, Manganotti P, Fiaschi A, et al. 2004. Modulation of motor cortex excitability after upper limb immobilization. Clin Neurophysiol, 115:1264-75.

Zanette G, Tinazzi M, Bonato C, et al. 1997. Reversible changes of motor cortical outputs following immobilization of the upper limb. Electroencephalogr Clin Neurophysiol, 105:269-79.

Ziemann U, Hallett M, Cohen LG. 1998. Mechanisms of deafferentationinduced plasticity in human motor cortex. J Neurosci, 18:7000-7. 